\title{
Angiogenic Signalling Pathways Altered in Gliomas: Selection Mechanisms for More Aggressive Neoplastic Subpopulations with Invasive Phenotype
}

\author{
Susana Bulnes, ${ }^{1}$ Harkaitz Bengoetxea, ${ }^{2}$ Naiara Ortuzar, ${ }^{2}$ Enrike G. Argandoña, ${ }^{3}$ \\ Álvaro Garcia-Blanco, ${ }^{2}$ Irantzu Rico-Barrio, ${ }^{2}$ and José V. Lafuente ${ }^{2}$ \\ ${ }^{1}$ Laboratory of Clinical and Experimental Neuroscience (LaNCE), Department of Nursing I, \\ University of the Basque Country, 48940 Leioa, Spain \\ ${ }^{2}$ Laboratory of Clinical and Experimental Neuroscience (LaNCE), Department of Neuroscience, \\ University of the Basque Country, Leioa, P.O. Box 699, 48080 Bilbao, Spain \\ ${ }^{3}$ Unit of Anatomy, Department of Medicine, University of Fribourg, 1700 Fribourg, Switzerland \\ Correspondence should be addressed to José V. Lafuente, onplasav@lg.ehu.es
}

Received 7 February 2012; Accepted 22 May 2012

Academic Editor: Laura Cerchia

Copyright (C) 2012 Susana Bulnes et al. This is an open access article distributed under the Creative Commons Attribution License, which permits unrestricted use, distribution, and reproduction in any medium, provided the original work is properly cited.

The angiogenesis process is a key event for glioma survival, malignancy and growth. The start of angiogenesis is mediated by a cascade of intratumoural events: alteration of the microvasculature network; a hypoxic microenvironment; adaptation of neoplastic cells and synthesis of pro-angiogenic factors. Due to a chaotic blood flow, a consequence of an aberrant microvasculature, tissue hypoxia phenomena are induced. Hypoxia inducible factor 1 is a major regulator in glioma invasiveness and angiogenesis. Clones of neoplastic cells with stem cell characteristics are selected by HIF-1. These cells, called "glioma stem cells" induce the synthesis of vascular endothelial growth factor. This factor is a pivotal mediator of angiogenesis. To elucidate the role of these angiogenic mediators during glioma growth, we have used a rat endogenous glioma model. Gliomas induced by prenatal ENU administration allowed us to study angiogenic events from early to advanced tumour stages. Events such as microvascular aberrations, hypoxia, GSC selection and VEGF synthesis may be studied in depth. Our data showed that for the treatment of gliomas, developing anti-angiogenic therapies could be aimed at GSCs, HIF-1 or VEGF. The ENU-glioma model can be considered to be a useful option to check novel designs of these treatment strategies.

\section{Introduction}

Gliomas are the most common type of primary tumour in the central nervous system. Glioblastomas (GBM) are the malignant form of gliomas (World Health Organization Grade IV), and progress from a lower-grade glioma (secondary GBM) or appear de novo without any preceding tumour (primary GBM). Glioblastomas are strongly angiogenic tumours displaying a high degree of vascular proliferation and endothelial hyperplasia. It is a neoplasia of glial lineage with high proliferative and invasive capacity and may spread to occupy an entire lobe or even a hemisphere of the brain [1].

The growth of the glioblastoma is related to vascular network adaptation due to the increase of the metabolic necessities of neoplastic cells. During the early stages of gliomas, the metabolic demand is supplied by the vast microvasculature of the CNS; however, when the metabolic supply capacity is exceeded during neoplastic progression, new formation of vessels becomes necessary [2-4]. The genesis of new vessels from preexisting ones is called angiogenesis, in opposition to vasculogenesis, which refers to the formation of vessels from hematopoietic niches $[5,6]$.

Angiogenesis is a complex phenomenon, necessary for the progression of malignant gliomas [7]. The start of the angiogenesis process requires some angiogenic exogenous stimulus, such as hypoxia, current metabolic requirements, and tumour growth. More than 25 different growth factors and cytokines related to the induction of angiogenesis have been reported [8]. The production of these proangiogenic factors is a result of genetic alterations or is induced by hypoxia. 
Intratumour hypoxia occurs at the time when there is an imbalance between oxygen supply and demand, due to irregular and chaotic blood flow [9]. This situation triggers the synthesis of proangiogenic factors such as matrix metalloprotease (MMP-2), angiopoietin-1, phosphoglycerate kinase (PGK), erythropoietin (EPO), and vascular endothelial growth factor (VEGF)-A [10]. Some of these are mediated by the hypoxia inducible factor (HIF-1). The increase of proangiogenic factors induces the start of angiogenesis, also known as the "angiogenic switch." This is a key moment for tumour malignancy, a critical step for the formation of new blood vessels and for the adaptation of the microenvironment to the growth of gliomas [11-13].

Vascular endothelial growth factor (VEGF) is the major regulator of angiogenesis in development [14-16] and disease [17-19]. VEGF-A triggers the angiogenic switch [4], reaching the target cells (endothelial cells) that express VEGF receptors [20]. However, the role of VEGF in nervous tissue is even more extensive. Previous studies have shown that VEGF also has strong neuroprotective, neurotrophic and neurogenic properties [11, 21-23]. The synthesis of VEGF is mediated by hypoxia inducible factor (HIF-1). Recently, researchers have reported that some neoplastic cells with stem cell characteristics called "glioma stem cells" play a pivotal role, inducing angiogenesis via HIF-1/VEGF [24]. Hypoxia has been related to clone selection of tumour cells. Clones adapted to the tumour microenvironment have acquired the phenotype of tumour stem cells, with increased proliferative and infiltrative capacity $[25,26]$. Invasion of adjacent normal parenchyma has also been attributed to glioma stem cells.

The onset of angiogenesis is mediated by a cascade of intratumoural events: alteration of the microvasculature network; a hypoxic microenvironment; adaptation of neoplastic cells; synthesis of proangiogenic factors,; finally the generation of new vessels. This chronological sequence of events is difficult to study during human glioma development. In order to follow the natural development of glioma pathogenesis, an endogenous glioma in rats was generated by transplacental administration of the carcinogen ethylnitrosourea (ENU) [27-30]. It has been reported that ENU gliomas are a representative model for human gliomas due to their location and also to their similar molecular and genetic alterations [31]. According to our experience, ENUgliomas have proven to be a useful tool in the study of many aspects of tumourigenesis and neoangiogenesis. This model permits various neoplastic stages to be isolated, to study the microvascular aberration process, the role of the proangiogenic cytokine VEGF and the glioma stem cells. This knowledge will contribute to define modern targets for anti-tumoural therapies.

\section{Vascular Endothelial Growth Factor (VEGF)}

Angiogenesis is a complex process that requires proteolytic and mitogenic activity of endothelial cells and interaction of these with the extracellular matrix molecules and periendothelial support cells (pericytes and smooth muscle cells). Numerous molecules and pathways are involved in this process, such as HIF-1, VEGF-A, its receptors VEGFR-1 and VEGFR-2, the endothelial receptor tyrosine kinase tie-1 and tie-2, and the angiopoietin ligands 1 and 2. Many other molecules, such as PDGF and TGF-B, integrin receptors, and so forth play a very important role.

The VEGF family consists of seven different homologous factors, VEGF-A, VEGF-B, VEGF-C, VEGF-D, VEGF-E, VEGF-F, and placental growth factor (PIGF) [32]. VEGF-A (VEGF) is the predominant form and is a hypoxia-inducible $45 \mathrm{KDa}$ homodimeric glycoprotein. It has five different isoforms, $\mathrm{VEGF}_{123}, \mathrm{VEGF}_{145}, \mathrm{VEGF}_{165}, \mathrm{VEGF}_{189}$ and $\mathrm{VEGF}_{206}$, that are produced by alternative splicing. Expression of VEGF-A was initially detected in a variety of tumour cell lines, while its receptors VEGFR-1 and VEGFR-2 were predominantly expressed in endothelial cells [33].

VEGF-A acts as a mitogenic, survival, and antiapoptotic factor for the endothelial cells from arteries, veins, and lymph vessels. Faced with increased secretion of VEGF-A and its binding to receptors on the surface of endothelial cells, VEGF-A is a signal transducer leading the production of molecules that include enzymes for the degradation of the extracellular matrix and for increase vascular permeability.

VEGF-A stimulates endothelial cell (EC) migration, proliferation, survival, permeability and lumen formation. Dvorak [34] described it as vascular permeability factor (VPF) due to its ability to induce leakage through the blood brain barrier (BBB) in some pathological situations $[35,36]$. Helmlinger et al. [37] stated that in the vasodilation process, VEGF induced the elongation of endothelial cells but not their proliferation. In the angiogenesis process, VEGF works alongside other factors such as angiopoietin and ephrins [38].

The synthesis of this proangiogenic cytokine has been described in neurons, astrocytes, pericytes, smooth muscle cells, macrophages, lymphoid cells, platelets, and fibroblasts [39]. In tumours, its synthesis is associated with neoplastic and endothelial cells of aberrant microvessels [17]. The VEGF-A gene is hypoxia regulated, due to a binding site for HIF in the promoter [40]. The rapid proliferation of the tumour, accompanied by a poor blood flow, leads to a relatively hypoxic environment in different areas of the tumour [40], resulting in upregulation of VEGF-A.

VEGF-A expression has been profusely reported in samples obtained from human gliomas [3]. In human glioma biopsies, it has been shown that VEGF-A overexpression correlates directly to proliferation, vascularization, and degree of malignancy, and therefore corresponds inversely to prognosis $[18,19,41]$. The material studied from human biopsies corresponds to advanced stages of tumour development with an adapted microvascular network. However, little is known about VEGF-A expression during glioma progression, especially during the early stages.

\section{Hypoxia}

During glioma progression, microvessels acquire aberrant morphologies, becoming tortuous, irregular and dilated, and displaying vascular leakage $[42,43]$. Consequently, the regional blood flow is irregular and chaotic and the 
functional deficit of blood perfusion induces intratumour hypoxia and ischemia [43]. Recent evidence suggests that a vascular collapse may precede angiogenesis in the development of the glioma. The vascular collapse leads to the death of neighbouring tumour cells and the formation of necrotic areas. In these regions, tissue hypoxia triggers the expression of HIF-1 and consequently VEGF-A, which initiates angiogenesis $[44,45]$.

Hypoxia is a critical aspect of the glioma microenvironment [46], and it has been associated with poor prognosis, increased angiogenesis, tumour growth, radio-and chemotherapy resistance, and tumour invasiveness $[47,48]$. It was observed that HIF-1 was involved in the oxygendependent expression of many genes, including those for pro-angiogenic and vascular permeability factors such us: VEGF [12, 13, 49], endothelial nitric oxide synthase (eNOS), angiopoietin, ephrin $[45,50]$, and others such as: glycolytic enzymes, glucose transporter-1 (Glut-1), transferrin, inducible nitric oxide synthase and heme oxigenase-1 [51], and TWIST [52]. In addition to oxygen levels, HIF-1 expression can be affected by several mechanisms, including the activation of oncogenes such as EGFR or loss of tumour suppressors, like p53 or PTEN [40].

HIF-1 is a heterodimeric transcriptional factor composed of two subunits, HIF- $1 \alpha$ and HIF- $1 \beta$. The HIF- $1 \beta$ subunit is constitutively expressed, whereas the HIF- $1 \alpha$ subunit is regulated by oxygen. The subunit HIF- $1 \alpha$ was originally discovered in 1988 as a unifying factor to the $3^{\prime}$ hypoxiainducible gene for erythropoietin (EPO). There are three isoforms of the alpha subunit: HIF $1 \alpha$, HIF $2 \alpha$ (also known as endothelial PAS-domain protein 1, EPAS1), and HIF3 $\alpha$ [53]. HIF $1 \alpha$ is responsible for the majority of the hypoxia response in cancer. Recently, a link has been discovered between HIF$1 \alpha$, tumour apoptosis, and the necrosis process [54].

\section{Glioma Stem Cells}

It has been reported that the hypoxic microenvironment plays a fundamental role in the induction of the neoplastic phenotype [55-57]. Some studies elucidated that HIF-2 $\alpha$ was only significantly present in the cancer stem cells, whereas HIF- $1 \alpha$ was present in both stem and nonstem tumour cells $[56,57]$.

Recently, a relationship between HIF- $1 \alpha$, tumour migration and stem cell biology has been found [47, 58]. Furthermore, studies of human GBM have described the relationship between the intratumour oxygen gradient and the appearance of tumour stem cells [59]. These cells, called "glioma stem cells" (GSCs), are thought to be responsible for the process of infiltration and subsequent tumour recurrence. Moreover, it has been reported that the relationship between angiogenesis and tumour invasiveness is due to this neoplastic subpopulation. Recently, researchers have reported that GSCs play a pivotal role in inducing angiogenesis via HIF-1/VEGF $[24,60]$.

Due to the regulatory role of GSCs in tumour angiogenesis, they are currently being considered as a potential antiangiogenic target. It is a fact that nowadays, research in novel antitumour therapies has centred on identifying these neoplastic cells. So far, markers have been used: CD133/Promonin-1, a prominin family of pentaspan membrane proteins; Nestin, a protein found in neural stem cells in SVZ and other markers of neuroepithelial stem cells: Musashi-1, Sox-2, GFAP, Map-2, Neural-tubulin, Neurofilament O4, Noggin, and CD15 have all been proposed in order to identify tumour stem cells [61-63].

In human glioblastoma, GSCs were identified by CD133 expression and associated with bad prognosis of the tumour $[64,65]$. However, little is known about their genesis during glioma progression, especially during the early stages.

\section{An Experimental Model of Endogenous Glioma}

As has previously been described, the onset of angiogenesis is mediated by a cascade of intratumoural events, where both tumour and "non-tumour" cells as well as numerous cytokines are involved. In order to follow the angiogenesis process during the neoplasia genesis, we have used an endogenous glioma model. It was generated by transplacental administration of ethylnitrosourea (ENU) in rats $[28,66$, 67].

Previous studies have reported that administration of ENU to adult rodents did not induce CNS tumours, while transplacental administration during the perinatal period (last week of gestation and first week of postnatal time) induced tumours in more than half of the offspring [68]. This model has allowed the study of some aspects related to tumour behaviour or possible treatment strategies in vivo [69].

This model shows that after the exposure of pregnant Sprague Dawley rats on the 15th day of gestation to a single intraperitoneal injection of $80 \mathrm{mg} \mathrm{ENU} / \mathrm{kg}$ body weight, more than half of the offspring developed intracerebral tumours, diagnosed as gliomas [17, 27]. According to histopathological features, immunophenotyping, proliferation index for Ki-67, and MRI study, we have demonstrated that this model reproduces quite closely the neuropathology of oligodendrogliomas and glioblastoma $[31,70]$.

Three glioma development stages were identified: early (microtumours), intermediate (the smallest macrotumours), and advanced stages (corresponding to the largest macrotumours) (Figure 1). Microtumours corresponding with cell proliferation mass and minor tumour node (Figure 1(a)) were diagnosed as low-degree gliomas, and macrotumours as anaplastic gliomas or high-degree gliomas (Figure 1(b)). The largest macrotumours corresponded to highly infiltrative malignant gliomas (Figure 1(c)). They displayed characteristic features of glioblastomas, such as haemorrhages, microvascular proliferation, macrocysts and necrosis with pseudo-palisades $[1,17]$. These results corroborate the origin of secondary GBM from oligodendrogliomas, and not only from astrocytoma cells.

ENU-tumour development shows that malignancy degree is related to rat age and consequently to the glioma size. In a fashion similar to human glioma pathology, the neoplasm has to reach a symptomatic size. At the age of 

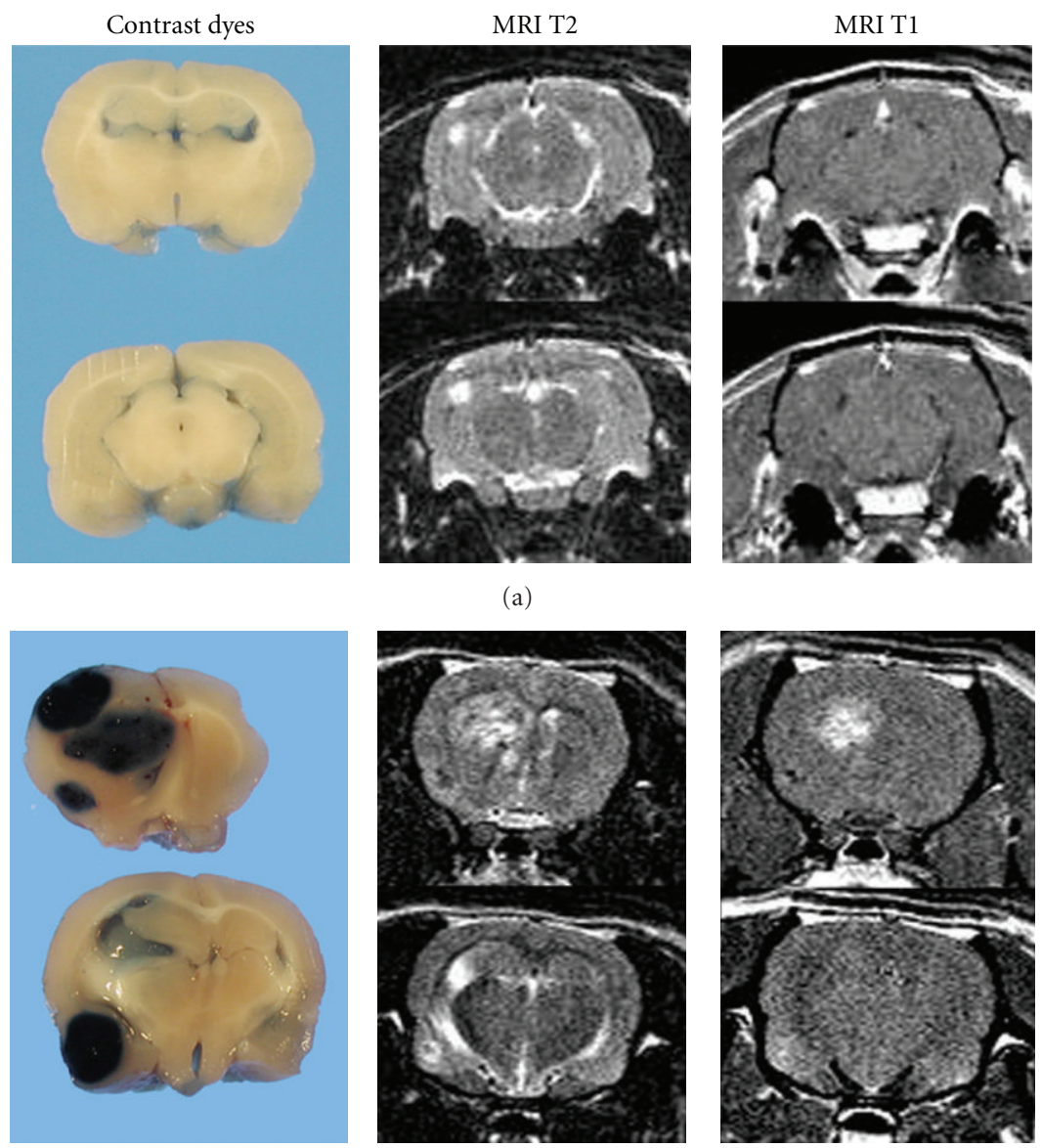

(b)
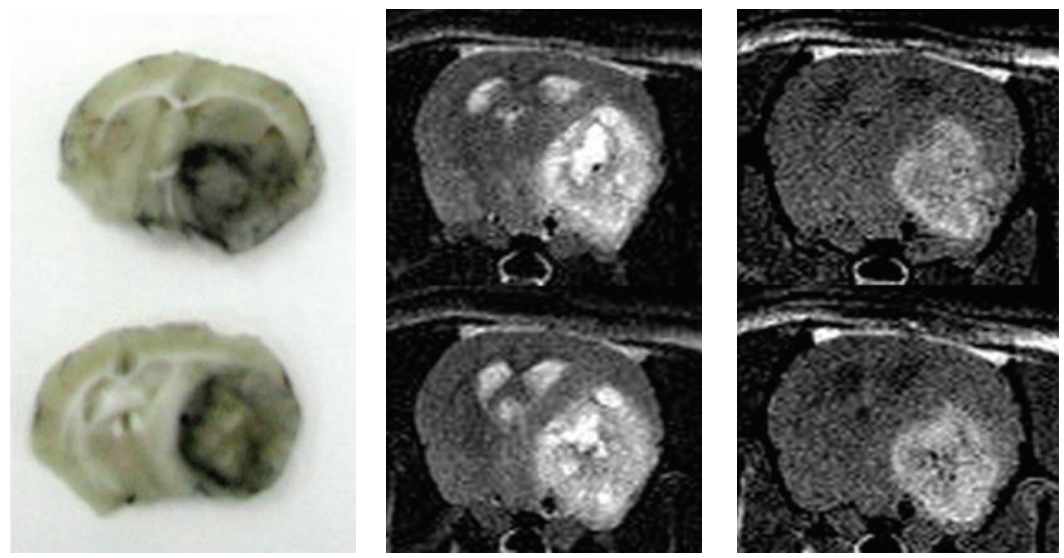

(c)

Figure 1: Brain coronal sections of Sprague Dawley rats exposed prenatally to ethylnitrosourea. Two columns show MRI on T1-w and T2$\mathrm{w}$ after gadolinium administration and the left column shows the necropsy thirty minutes after Evans Blue i.v. injection (a, b) or Indian ink (c). (a) On T2-weighted images, tumours in the early development stage show a diminutive proliferation mass growing in association with the subcortical white matter. The blood brain barrier (BBB) remains still intact, shown by the lack of contrast or dye extravasation (microtumours). (b) Multiple tumours on the intermediate development stage. There is a BBB dysfunction indicated by extravasation of Evans Blue and by gadolinium enhancing the contrast on T1 images (macrotumours). (c) A macrotumour in the advanced stage growing over a whole hemisphere. It displays a heterogeneous signal on T2 and on T1 due to the presence of histopathological features of malignancy such as haemorrhages, cysts, and necrosis. With Indian ink, brains show in black a ring of aberrant vessels surrounding the neoplasia. The same typical shape of glioblastoma multiforme may be observed on MRI in T1 with gadolinium. 


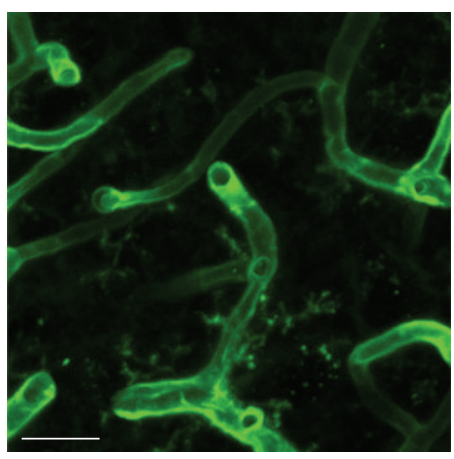

(a)

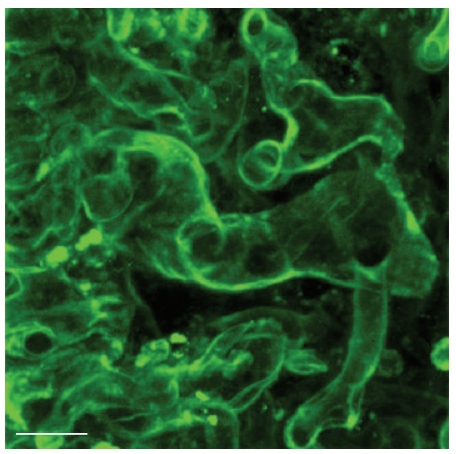

(c)

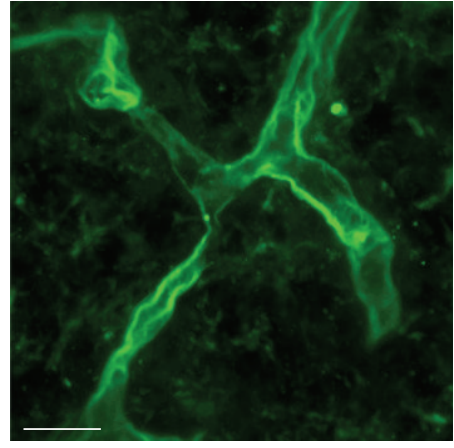

(b)

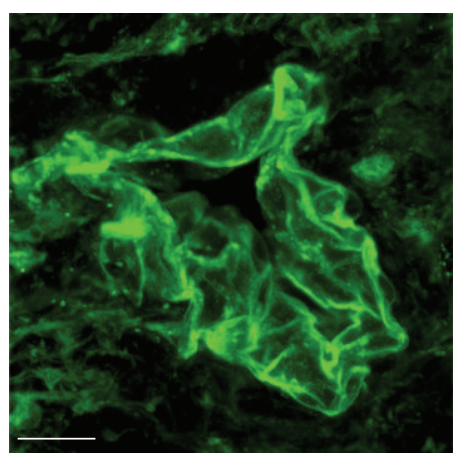

(d)

FIGURE 2: Confocal microphotographs of the microvascular network during development of an ENU-induced glioma. Histochemistry for tomato lectin (LEA-FITC) performed on $40 \mu \mathrm{m}$ sections. (a) Similar angioarchitecture to the normal brain during the early stage. (b) Tortuous and dilated vessels during the intermediate development stage. (c) The microvascular network is mainly composed of glomeruloid vessels in the periphery area, (d) Occasional huge dilated vessels in the intratumour region (bar scale of $20 \mu \mathrm{m}$ ).

four months, proliferations of oligodendrocytes in the subcortical white matter are frequently seen. These proliferations become nodular at six months, although the rats did not display symptoms. These nodes grow and in one year may occupy a half hemisphere, or extend even more toward the contralateral hemisphere. These macrotumours may display clinical signs, depending on size and malignancy.

Small tumours, characteristic of the early phase, remain asymptomatic and undetected due to their small size, therefore, they are named microtumours. Several authors have considered these noninvasive tumours as dormant tumours. The existence of dormant tumours has notable implications for the early detection and treatment of cancer [71].

\section{Glioma Hypoxic Microenvironment}

In primary tumours, the dormancy state is best defined as the time between carcinogenic transformation and the onset of inexorable progressive growth. There are several mechanisms related to the emergence from tumour latency, and one of them is the induction of angiogenesis. The escape of tumours from dormancy depends on the cell population undergoing an angiogenic switch that induces the neoformation of functional vessels [72].

Prior to angiogenesis and to the acquisition of the tumour stem cell phenotype, events like microvascular aberration and subsequent intratumour hypoxia take place. The tumour microvascular network suffers some adaptations to the current requirements of the tumour. The ENUmodel allows microvascular changes to be followed before angiogenesis. The adaptive changes of the microvasculature from nonangiogenic gliomas (microtumours) to angiogenic gliomas (macrotumours) involve an increase in the tumour area occupied by the vascular network and a decrease in vascular density [73]. We have demonstrated a transition from the homogeneous capillary network of early stages to an anarchic angioarchitecture in advanced stages (Figure 2). It was found that the vessel density decreased and the vascular size increased with increased malignancy [70]. ENU-gliomas in the initial stage show microvessels very similar to normal brain capillaries (Figure 2(a)), in the intermediate stage they are already tortuous, disorganized, and dilated (Figure 2(b)), and in the advanced stage they become anarchic and aberrant with topographies such as multilayered "glomeruloid tufts", "garlands" of proliferated vessels (Figure 2(c)) and huge dilated vessels (Figure 2(d)) [70].

Microvascular adaptations in early development stages were based on vasodilatation, endothelium elongation, and permeability increase mediated by VEGF without BBB dysfunction [70]. In malignant gliomas the microvascular adaptations vary according to blood flow perfusion. Permeability increase in intratumour vessels is not enough to supply the metabolic demand, and triggering of the angiogenesis process on the tumour border is necessary. 


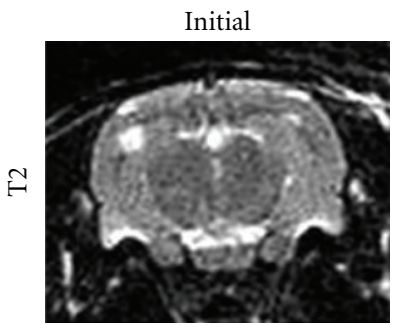

(a)

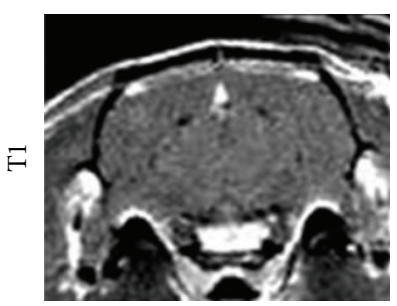

(d)

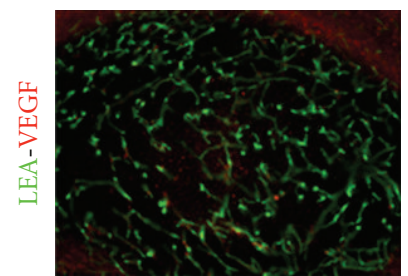

(g)

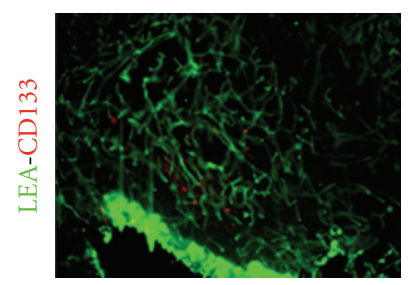

(j)

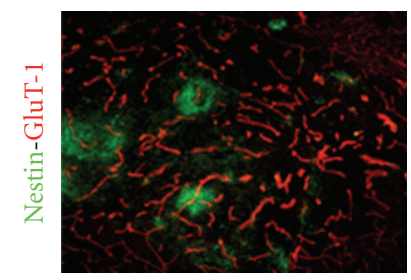

$(\mathrm{m})$

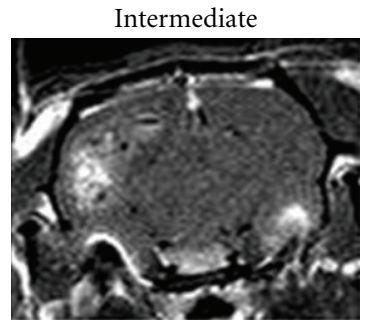

(b)

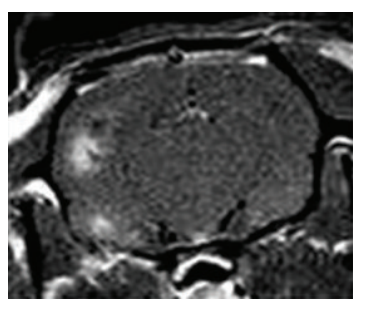

(e)

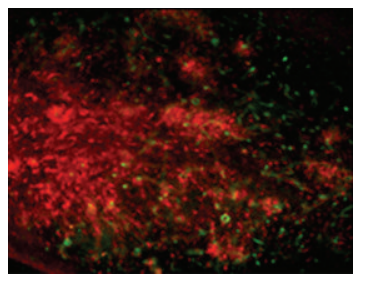

(h)

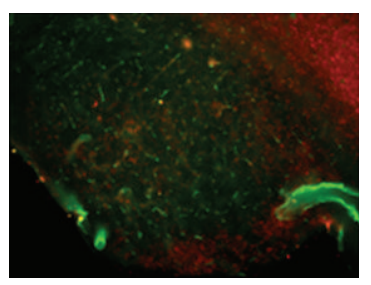

(k)

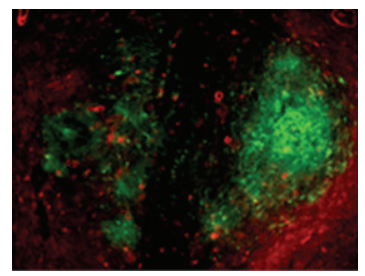

(n)

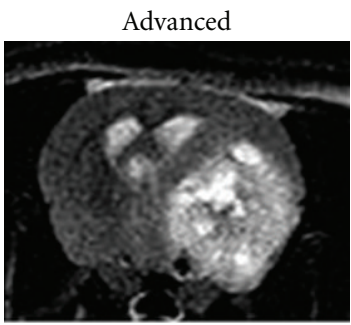

(c)

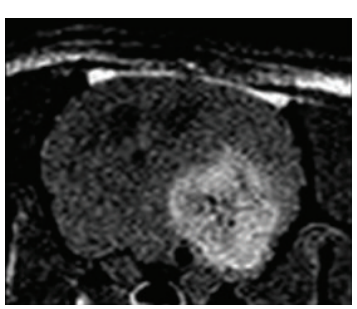

(f)

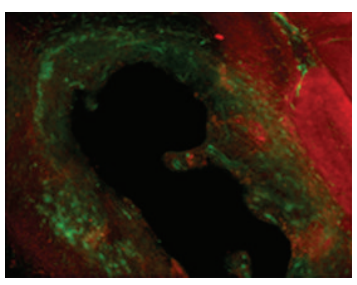

(i)

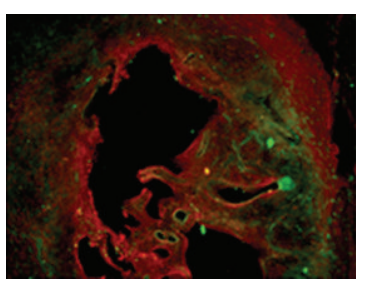

(1)

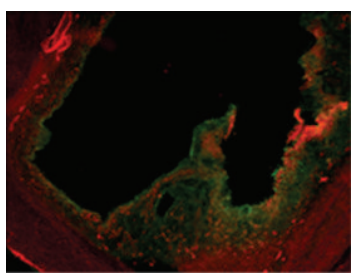

(o)

FIGURE 3: Expression of stem cell markers: CD133 (red) and Nestin (green), proangiogenic factor VEGF165 (red) and microvasculature markers: LEA lectin (green) and GluT-1 (red) during the ENU-glioma development. (a-f) MRI on T2-w and T1-w after gadolinium injection. (a, d) During the initial stage, the ENU-glioma grows in association with the subcortical white matter. It shows a homogeneous hyperintense signal on T2-w (a) and an isointense signal on T1-w (d). (b) ENU-intermediate stage corresponds with the "angiogenic switch." (c, f) ENU-Glioblastoma displays heterogeneous hyperintense signal on T2 and on T1-w. (g-h) Overexpression of VEGF165 is shown in the intermediate ( $\mathrm{h}$ ) and advanced stages. (i) Also shown in perivascular cells of glomeruloid vessels. ( $\mathrm{j}-\mathrm{l}$ ) CD133 expression is found following the intermediate stage (k). (l) ENU-GBM displays plenty of CD133+ cells in the intratumour hypoxic area and bordering the tumour. (m-o) Nestin+ cells are detected in every ENU development stage. Nestin+ cells are grouped into intratumour niches and around the microvessels. (Immunofluorescence images at 100x amplification, except I, l, and o at 40x). 


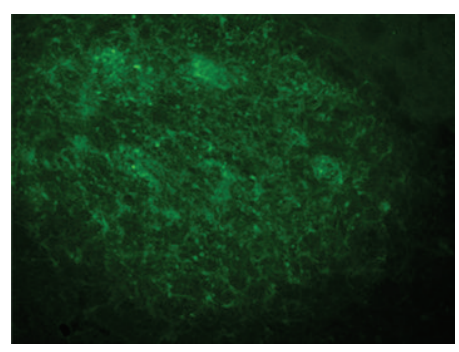

(a)

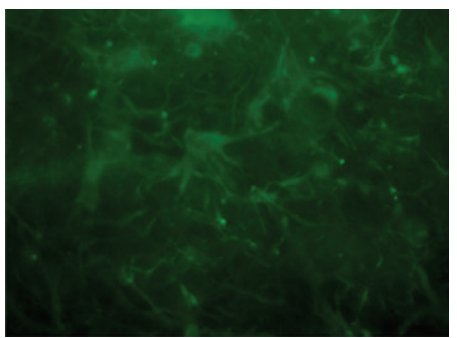

(d)

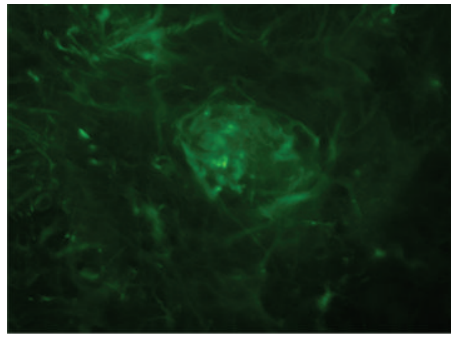

(g)

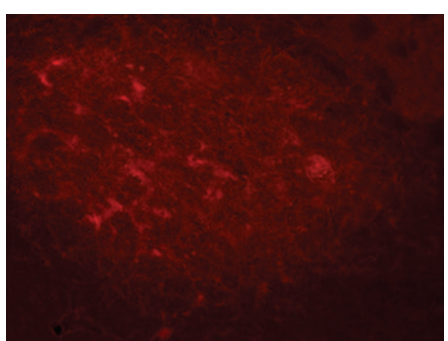

(b)

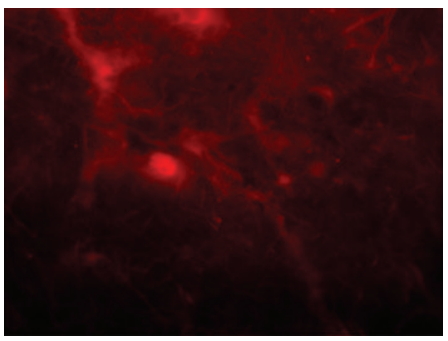

(e)

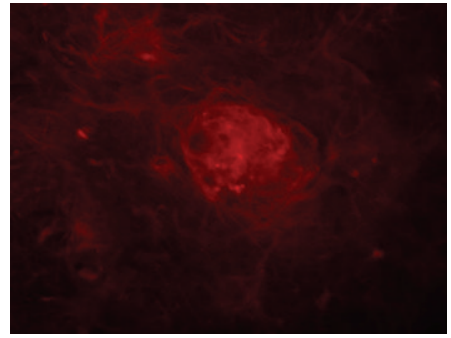

(h)

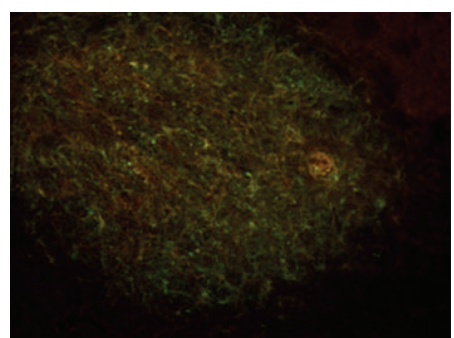

(c)

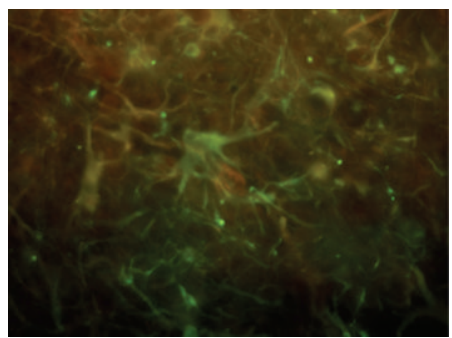

(f)

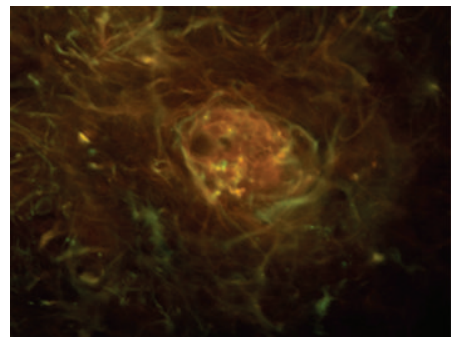

(i)

Figure 4: Intermediate ENU glioma stage showing the expression of Nestin (green) and CD133 (red) by double immunofluorescence. (a-c) Intratumour niches of glioma stem cells positive for Nestin (a) and CD133 (b). (c) Both stem cell markers, Nestin and CD133 are coexpressed in some of these cells. (d-f) Isolated cells in the border of the tumour with a shape similar to astrocytes. Although the majority of these cells are Nestin-CD133 positive, there is a greater density of Nestin + cells than CD133+ ones. (g-i) Perivascular niche of glioma stem cells displaying colocation of both markers.

\section{The Angiogenic Switch}

The transition from a prevascular proliferative node to a highly vascularised and progressively outgrowing tumour is referred to as the "angiogenic switch" [74]. Angiogenesis is activated in growing gliomas when the proangiogenic stimuli outweigh the antiangiogenic stimuli. This moment is detected in the ENU-glioma intermediate stage by the presence of overexpression of VEGF and eNOS [75] (Figure 3). In this phase, hypoxia increases VEGF and eNOS expression through the activation of the PI-K/Akt cascade $[76,77]$.

In this intermediate stage, we identified glioma stem cells by CD133 and Nestin immunostaining (Figure 4). We found that some cells coexpress the Nestin, CD133 and VEGF165 antibodies. These cells were distributed in intratumour niches and perivascular niches around the tortuous and aberrant vessels (intermediate-advanced stages). The distribution of CD133+ cells corresponded mainly to overexpression of VEGF in neoangiogenic border areas and intratumour hypoxic areas of neoplasia $[17,70,78]$. It has been reported that tumour stem cells over-express VEGF, so this cell population could be involved in the process of angiogenesis.
Our results agree with the staining of CD133 described in the advanced and intermediate stages of human gliomas. Therefore, CD133 expression has been related to poor prognosis [79].

In the ENU model, as in human GBMs, the angiogenic factor VEGF-A and HIF- $1 \alpha$ have been immunohistochemically identified in cells located inside the pseudopalisade area around necrosis. This feature provides a link between hypoxia and angiogenesis in malignant gliomas [13, 39] (unpublished data). In addition, we found Nestin+ and CD133+ cells around necrotic areas as well [78]. These findings support the idea that intratumour hypoxia contributes significantly to the selection of glioma stem cells (GSCs) and furthermore these groups of cells could be responsible for the infiltrative process and neoplastic recurrences $[9,56]$.

\section{Neovasculature and Tumour Invasiveness}

Tumour microvasculature generates specific microenvironments that promote the formation and/or maintenance of brain GSCs. New vessels often show immature walls that are inherently leaky. Tissue may become oedematous. In 
previous studies, the neoangiogenic area of these tumours was replete with small vessels overexpressing VEGF and displaying an irregular staining for BBB markers [70]. It is a fact that, even though brain tumour vessels appear more "leaky" than their normal tissue counterpart, at least some elements of the $\mathrm{BBB}$ in glioblastoma remain intact. Permeability is varied, and the heterogeneity of permeability contributes to uneven distribution of transport products, such us oxygen and chemotherapeutic drugs within the tumour $[80]$.

It has also been proposed that the abnormal blood vessels in gliomas create a vascular niche that houses glioma stem cells $[81,82]$. This "perivascular niche" consists of a rich extracellular matrix (ECM) and nontumoural cells, which provide a physical support for tissue architecture. There is consistent evidence about the migration of GSCs using the ECM $[83,84]$. If the recruitment of aberrant vascular niches is a critical component in the progression of brain tumours, this might explain why the most aggressive brain tumours are highly angiogenic $[19,85]$. Moreover, VEGF isoforms are at least partially bound to the ECM, and their degradation could provide an extra source of growth factors [86].

Calabrese and colleagues [81] showed that endothelial cells (ECs) interact closely with brain tumour stem cells in the perivascular location (termed the perivascular niche) and secrete factors that maintain these cells in a stem cell-like state. Moreover, recent studies of orthotopic glioblastoma xenografts suggested that GSCs of these brain tumours secrete angiogenic factors that promote the recruitment and formation of tumour blood vessels [24].

Folkins and colleagues [60] compared the angiogenesis in tumour xenografts from C6 glioma cells containing either a low or a high fraction of GSCs and found that GSC-high xenograft tumours demonstrated an increased microvessel density and blood perfusion.

\section{Conclusion}

Glioma survival and growth is dependant on angiogenesis. Angiogenesis is a complex process in which several cellular and molecular pathways are involved. Among the cellular pathways, glioma stem cells and vascular endothelial cells play a relevant role, and among the molecular pathways, hypoxia inducible factor -1 (HIF-1) and vascular endothelial growth factor (VEGF) are the most significant. Both molecular mediators are related to glioma stem cells. Therefore, hypoxia selects the GSCs that induce the start of the angiogenesis process by the synthesis of proangiogenic factors such a angiopoietin, ECM factors and VEGF, and decrease angiostatic factors [87]. On the other hand, GSCs mediate vascular adaptation not only by the synthesis of vascular permeability factors or angiogenic factors, but also by differentiation in endothelial cells of the neovascular network.

\section{Acknowledgments}

This paper has been partially supported by SAIOTEK and GIC 491/10 Basque Government.

\section{References}

[1] P. Kleihues, P. C. Burger, K. D. Aldape et al., "Glioblastoma," in WHO Classification of Tumours of the Central Nervous System, D. N. Louis, H. Ohgaki, O. D. Wiestler, and W. K. Cavenee, Eds., pp. 33-49, Agency for Research on Cancer (IARC), Lyon, France, 2007.

[2] G. Bergers and L. E. Benjamin, "Tumorigenesis and the angiogenic switch," Nature Reviews Cancer, vol. 3, no. 6, pp. 401410, 2003.

[3] P. Carmeliet and R. K. Jain, "Angiogenesis in cancer and other diseases," Nature, vol. 407, no. 6801, pp. 249-257, 2000.

[4] G. D. Yancopoulos, S. Davis, N. W. Gale, J. S. Rudge, S. J. Wiegand, and J. Holash, "Vascular-specific growth factors and blood vessel formation," Nature, vol. 407, no. 6801, pp. 242248, 2000.

[5] P. Carmeliet, "Angiogenesis in health and disease," Nature Medicine, vol. 9, no. 6, pp. 653-660, 2003.

[6] W. Risau, "Mechanisms of angiogenesis," Nature, vol. 386, no. 6626, pp. 671-674, 1997.

[7] J. Folkman, "Angiogenesis," Annual Review of Medicine, vol. 57, pp. 1-18, 2006.

[8] M. L. H. Wong, A. Prawira, A. H. Kaye, and C. M. Hovens, "Tumour angiogenesis: its mechanism and therapeutic implications in malignant gliomas," Journal of Clinical Neuroscience, vol. 16, no. 9, pp. 1119-1130, 2009.

[9] R. L. Jensen, "Hypoxia in the tumorigenesis of gliomas and as a potential target for therapeutic measures," Neurosurgical Focus, vol. 20, no. 4, p. E24, 2006.

[10] G. H. Fong, "Mechanisms of adaptive angiogenesis to tissue hypoxia," Angiogenesis, vol. 11, no. 2, pp. 121-140, 2008.

[11] K. Jin, Y. Zhu, Y. Sun, X. O. Mao, L. Xie, and D. A. Greenberg, "Vascular endothelial growth factor (VEGF) stimulates neurogenesis in vitro and in vivo," Proceedings of the National Academy of Sciences of the United States of America, vol. 99, no. 18, pp. 11946-11950, 2002.

[12] H. J. H. Marti, M. Bernaudin, A. Bellail et al., "Hypoxiainduced vascular endothelial growth factor expression precedes neovascularization after cerebral ischemia," American Journal of Pathology, vol. 156, no. 3, pp. 965-976, 2000.

[13] G. L. Semenza, "Defining the role of hypoxia-inducible factor 1 in cancer biology and therapeutics," Oncogene, vol. 29, no. 5, pp. 625-634, 2010.

[14] H. Bengoetxea, E. G. Argandoña, and J. V. Lafuente, "Effects of visual experience on vascular endothelial growth factor expression during the postnatal development of the rat visual cortex," Cerebral Cortex, vol. 18, no. 7, pp. 1630-1639, 2008.

[15] N. Ferrara, H. P. Gerber, and J. LeCouter, "The biology of VEGF and its receptors," Nature Medicine, vol. 9, no. 6, pp. 669-676, 2003.

[16] L. R. Ment, W. B. Stewart, R. Fronc et al., "Vascular endothelial growth factor mediates reactive angiogenesis in the postnatal developing brain," Developmental Brain Research, vol. 100, no. 1, pp. 52-61, 1997.

[17] S. Bulnes and J. V. Lafuente, "VEGF immunopositivity related to malignancy degree, proliferative activity and angiogenesis in ENU-induced gliomas," Journal of Molecular Neuroscience, vol. 33, no. 2, pp. 163-172, 2007.

[18] J. V. Lafuente, B. Adán, K. Alkiza, J. M. Garibi, M. Rossi, and F. F. Cruz-Sánchez, "Expression of vascular endothelial growth factor (VEGF) and platelet- derived growth factor receptor- $\beta$ (PDGFR- $\beta$ ) in human gliomas," Journal of Molecular Neuroscience, vol. 13, no. 1-2, pp. 177-185, 1999. 
[19] K. H. Plate, "Mechanisms of angiogenesis in the brain," Journal of Neuropathology and Experimental Neurology, vol. 58, no. 4, pp. 313-320, 1999.

[20] B. Millauer, S. Wizigmann-Voos, H. Schnurch et al., "High affinity VEGF binding and developmental expression suggest Flk-1 as a major regulator of vasculogenesis and angiogenesis," Cell, vol. 72, no. 6, pp. 835-846, 1993.

[21] N. Ortuzar, E. G. Argandoña, H. Bengoetxea, and J. V. Lafuente, "Combination of intracortically administered VEGF and environmental enrichment enhances brain protection in developing rats," Journal of Neural Transmission, vol. 118, no. 1, pp. 135-144, 2011.

[22] J. M. Rosenstein and J. M. Krum, "New roles for VEGF in nervous tissue-beyond blood vessels," Experimental Neurology, vol. 187, no. 2, pp. 246-253, 2004.

[23] E. Storkebaum, D. Lambrechts, and P. Carmeliet, "VEGF: once regarded as a specific angiogenic factor, now implicated in neuroprotection," BioEssays, vol. 26, no. 9, pp. 943-954, 2004.

[24] S. Bao, Q. Wu, R. E. McLendon et al., "Glioma stem cells promote radioresistance by preferential activation of the DNA damage response," Nature, vol. 444, no. 7120, pp. 756-760, 2006.

[25] J. M. Heddleston, Z. Li, R. E. McLendon, A. B. Hjelmeland, and J. N. Rich, "The hypoxic microenvironment maintains glioblastoma stem cells and promotes reprogramming towards a cancer stem cell phenotype," Cell Cycle, vol. 8, no. 20, pp. 3274-3284, 2009.

[26] Z. Li. Z., H. Wang, C. E. Eyler, A. B. Hjelmeland, and J. N. Rich, "Turning cancer stem cells inside out: an exploration of glioma stem cells signalling pathways," The Journal of Biological Chemistry, vol. 284, no. 25, pp. 16705-16709, 2009.

[27] S. Bulnes-Sesma, N. Ullibarri-Ortiz De Zárate, and J. V. Lafuente-Sánchez, "Tumour induction by ethylnitrosourea in the central nervous system," Revista de Neurologia, vol. 43, no. 12, pp. 733-738, 2006.

[28] P. E. Kish, M. Blaivas, M. Strawderman et al., "Magnetic resonance imaging of ethyl-nitrosourea-induced rat gliomas: a model for experimental therapeutics of low-grade gliomas," Journal of Neuro-Oncology, vol. 53, no. 3, pp. 243-257, 2001.

[29] P. L. Lantos, "Development of nitrosourea-induced brain tumours-with a special note on changes occurring during latency," Food and Chemical Toxicology, vol. 24, no. 2, pp. 121127, 1986.

[30] D. Schiffer, L. Annovazzi, V. Caldera, and M. Mellai, "On the origin and growth of gliomas," Anticancer Research, vol. 30, no. 6, pp. 1977-1998, 2010.

[31] D. M. Kokkinakis, E. J. Rushing, M. M. Shareef et al., "Physiology and gene expression characteristics of carcinogen-initiated and tumor-transformed glial progenitor cells derived from the CNS of methylnitrosourea (MNU)-treated Sprague-Dawley rats," Journal of Neuropathology and Experimental Neurology, vol. 63, no. 11, pp. 1182-1199, 2004.

[32] N. Ferrara, "Vascular endothelial growth factor: basic science and clinical progress," Endocrine Reviews, vol. 25, no. 4, pp. 581-611, 2004.

[33] F. S. Grünewald, A. E. Prota, A. Giese, and K. Ballmer-Hofer, "Structure-function analysis of VEGF receptor activation and the role of coreceptors in angiogenic signaling," Biochimica et Biophysica Acta, vol. 1804, no. 3, pp. 567-580, 2010.

[34] H. F. Dvorak, "Discovery of vascular permeability factor (VPF)," Experimental Cell Research, vol. 312, pp. 522-526, 2006.
[35] N. Ferrara, "The role of VEGF in the regulation of physiological and pathological angiogenesis," EXS, no. 94, pp. 209-231, 2005.

[36] J. V. Lafuente, S. Bulnes, B. Mitre, and H. H. Riese, "Role of VEGF in an experimental model of cortical micronecrosis," Amino Acids, vol. 23, no. 1-3, pp. 241-245, 2002.

[37] G. Helmlinger, M. Endo, N. Ferrara, L. Hlatky, and R. K. Jain, "Growth factors: formation of endothelial cell networks," $\mathrm{Na}$ ture, vol. 405, no. 6783, pp. 139-141, 2000.

[38] T. Tonini, F. Rossi, and P. P. Claudio, "Molecular basis of angiogenesis and cancer," Oncogene, vol. 22, no. 43, pp. 6549-6556, 2003.

[39] D. Zagzag, D. R. Friedlander, B. Margolis et al., "Molecular events implicated in brain tumor angiogenesis and invasion," Pediatric Neurosurgery, vol. 33, no. 1, pp. 49-55, 2000.

[40] B. Kaur, F. W. Khwaja, E. A. Severson, S. L. Matheny, D. J. Brat, and E. G. Van Meir, "Hypoxia and the hypoxia-inducible-factor pathway in glioma growth and angiogenesis," Neuro-Onco$\log y$, vol. 7, no. 2, pp. 134-153, 2005.

[41] L. D. Ke, Y. X. Shi, S. A. Im, X. Chen, and W. K. A. Yung, "The relevance of cell proliferation, vascular endothelial growth factor, and basic fibroblast growth factor production to angiogenesis and tumorigenicity in human glioma cell lines," Clinical Cancer Research, vol. 6, no. 6, pp. 2562-2572, 2000.

[42] P. Baluk, H. Hashizume, and D. M. M, "Cellular abnormalities of blood vessels as targets in cancer," Current Opinion in Genetics and Development, vol. 15, no. 1, pp. 102-111, 2005.

[43] H. Hashizume, P. Baluk, S. Morikawa et al., "Openings between defective endothelial cells explain tumor vessel leakiness," American Journal of Pathology, vol. 156, no. 4, pp. 1363 1380, 2000.

[44] J. Holash, P. C. Maisonpierre, D. Compton et al., "Vessel cooption, regression, and growth in tumors mediated by angiopoietins and VEGF," Science, vol. 284, no. 5422, pp. 1994-1998, 1999.

[45] D. Zagzag, H. Zhong, J. M. Scalzitti, E. Laughner, J. W. Simons, and G. L. Semenza, "Expression of hypoxia-inducible factor $1 \alpha$ in brain tumors: association with angiogenesis, invasion, and progression," Cancer, vol. 88, no. 11, pp. 2606-2618, 2000.

[46] B. Blouw, H. Song, T. Tihan et al., "The hypoxic response of tumors is dependent on their microenvironment," Cancer Cell, vol. 4, no. 2, pp. 133-146, 2003.

[47] O. Méndez, J. Zavadil, M. Esencay et al., "Knock down of HIF$1 \alpha$ in glioma cells reduces migration in vitro and invasion in vivo and impairs their ability to form tumor spheres," Molecular Cancer, vol. 9, article 133, 2010.

[48] R. Sullivan and C. H. Graham, "Hypoxia-driven selection of the metastatic phenotype," Cancer and Metastasis Reviews, vol. 26, no. 2, pp. 319-331, 2007.

[49] K. L. Jin, X. O. Mao, T. Nagayama, P. C. Goldsmith, and D. A. Greenberg, "Induction of vascular endothelial growth factor and hypoxia-inducible factor- $1 \alpha$ by global ischemia in rat brain," Neuroscience, vol. 99, no. 3, pp. 577-585, 2000.

[50] P. Vajkoczy, M. Farhadi, A. Gaumann et al., "Microtumor growth initiates angiogenic sprouting with simultaneous expression of VEGF, VEGF receptor-2, and angiopoietin-2," The Journal of Clinical Investigation, vol. 109, no. 6, pp. 777785, 2002.

[51] R. H. Wenger and M. Gassmann, "Oxygen(es) and the hypoxia-inducible factor-1," Biological Chemistry, vol. 378, no. 7, pp. 609-616, 1997. 
[52] M. H. Yang and K. J. Wu, "TWIST activation by hypoxia inducible factor-1 (HIF-1): implications in metastasis and development," Cell Cycle, vol. 7, no. 14, pp. 2090-2096, 2008.

[53] M. S. Wiesener, J. S. Jürgensen, C. Rosenberger et al., "Widespread hypoxia-inducible expression of HIF-2alpha in distinct cell populations of different organs," The FASEB Journal, vol. 17, no. 2, pp. 271-273, 2003.

[54] W. Chen, R. P. Ostrowski, A. Obenaus, and J. H. Zhang, "Prodeath or prosurvival: two facets of hypoxia inducible factor-1 in perinatal brain injury," Experimental Neurology, vol. 216, no. 1, pp. 7-15, 2009.

[55] C. E. Griguer, C. R. Oliva, E. Gobin et al., "CD133 is a marker of bioenergetic stress in human glioma," PLOS ONE, vol. 3, no. 11, Article ID e3655, 2008.

[56] J. M. Heddleston, Z. Li, J. D. Lathia, S. Bao, A. B. Hjelmeland, and J. N. Rich, "Hypoxia inducible factors in cancer stem cells," British Journal of Cancer, vol. 102, no. 5, pp. 789-795, 2010.

[57] Z. Li, S. Bao, Q. Wu et al., "Hypoxia-inducible factors regulate tumourigenic capacity of glioma stem cells," Cancer Cell, vol. 15, no. 6, pp. 501-513, 2009.

[58] E. E. Bar, A. Lin, V. Mahairaki, W. Matsui, and C. G. Eberhart, "Hypoxia increases the expression of stem-cell markers and promotes clonogenicity in glioblastoma neurospheres," American Journal of Pathology, vol. 177, no. 3, pp. 1491-1502, 2010.

[59] F. Pistollato, S. Abbadi, E. Rampazzo et al., "Intratumoral hypoxic gradient drives stem cells distribution and MGMT expression in glioblastoma," Stem Cells, vol. 28, no. 6, pp. 851$862,2010$.

[60] C. Folkins, Y. Shaked, S. Man et al., "Glioma tumor stem-like cells promote tumor angiogenesis and vasculogenesis via vascular endothelial growth factor and stromal-derived factor 1 ," Cancer Research, vol. 69, no. 18, pp. 7243-7251, 2009.

[61] P. Dell'Albani, "Stem cell markers in gliomas," Neurochemical Research, vol. 33, no. 12, pp. 2407-2415, 2008.

[62] T. Jang, N. S. Litofsky, T. W. Smith, A. H. Ross, and L. D. Recht, "Aberrant nestin expression during ethylnitrosourea-(ENU)induced neurocarcinogenesis," Neurobiology of Disease, vol. 15, no. 3, pp. 544-552, 2004.

[63] C. G. Hadjipanayis and E. G. Van Meir, "Brain cancer propagating cells: biology, genetics and targeted therapies," Trends in Molecular Medicine, vol. 15, no. 11, pp. 519-530, 2009.

[64] I. Germano, V. Swiss, and P. Casaccia, "Primary brain tumors, neural stem cell, and brain tumor cancer cells: where is the link?" Neuropharmacology, vol. 58, no. 6, pp. 903-910, 2010.

[65] T. N. Ignatova, V. G. Kukekov, E. D. Laywell, O. N. Suslov, F. D. Vrionis, and D. A. Steindler, "Human cortical glial tumors contain neural stem-like cells expressing astroglial and neuronal markers in vitro," Glia, vol. 39, no. 3, pp. 193-206, 2002.

[66] H. D. Mennel, N. Kosse, J. T. Heverhagen, and H. Alfke, "Primary and transplanted ENU induced rat tumors in neurooncology," Experimental and Toxicologic Pathology, vol. 56, no. 12, pp. 25-35, 2004.

[67] B. C. Zook and S. J. Simmens, "Neurogenic tumors in rats induced by ethylnitrosourea," Experimental and Toxicologic Pathology, vol. 57, no. 1, pp. 7-14, 2005.

[68] W. Slikker, N. Mei, and T. Chen, "N-ethyl-N-nitrosourea (ENU) increased brain mutations in prenatal and neonatal mice but not in the adults," Toxicological Sciences, vol. 81, no. 1, pp. 112-120, 2004.

[69] D. S. Beniashvili and V. N. Anisimov, "Morphology of experimentally induced tumors of the sympathetic nervous system in rats," Experimental and Toxicologic Pathology, vol. 56, no. 1-2, pp. 53-58, 2004.
[70] S. Bulnes, J. Bilbao, and J. V. Lafuente, "Microvascular adaptive changes in experimental endogenous brain gliomas," Histology and Histopathology, vol. 24, no. 6, pp. 693-706, 2009.

[71] N. Almog, "Molecular mechanisms underlying tumor dormancy," Cancer Letters, vol. 294, no. 2, pp. 139-146, 2010.

[72] N. Almog, L. Ma, R. Raychowdhury et al., "Transcriptional switch of dormant tumors to fast-growing angiogenic phenotype," Cancer Research, vol. 69, no. 3, pp. 836-844, 2009.

[73] P. Korkolopoulou, E. Patsouris, N. Kavantzas et al., "Prognostic implications of microvessel morphometry in diffuse astrocytic neoplasms," Neuropathology and Applied Neurobiology, vol. 28, no. 1, pp. 57-66, 2002.

[74] V. Baeriswyl and G. Christofori, "The angiogenic switch in carcinogenesis," Seminars in Cancer Biology, vol. 19, no. 5, pp. 329-337, 2009.

[75] S. Bulnes, E. G. Argandoña, H. Bengoetxea, O. Leis, N. Ortuzar, and J. V. Lafuente, "The role of eNOS in vascular permeability in ENU-induced gliomas," Acta Neurochirurgica. Supplement, vol. 106, pp. 277-282, 2010.

[76] N. M. Mazure, E. Y. Chen, K. R. Laderoute, and A. J. Giaccia, "Induction of vascular endothelial growth factor by hypoxia is modulated by a phosphatidylinositol 3-kinase/Akt signaling pathway in Ha-ras-transformed cells through a hypoxia inducible factor-1 transcriptional element," Blood, vol. 90, no. 9, pp. 3322-3331, 1997.

[77] S. Bulnes, Á. García-Blanco, H. Bengoetxea, N. Ortuzar, E. G. Argandoña, and J. V. Lafuente, "Glial stem cells and their relationship with tumour angiogenesis process," Revista de Neurologia, vol. 52, no. 12, pp. 743-750, 2011.

[78] S. Bulnes, H. Bengoetxea, N. Ortuzar, E. G. Argandon a, and J. V. Lafuente, "Endogenous experimental glioma model, links between glioma stem cells and angiogenesis," in GliomaExploring Its Biology and Practical Relevance, A. Ghosh, Ed., InTech, 2011.

[79] F. Zeppernick, R. Ahmadi, B. Campos et al., "Stem cell marker CD133 affects clinical outcome in glioma patients," Clinical Cancer Research, vol. 14, no. 1, pp. 123-129, 2008.

[80] M. C. Tate and M. K. Aghi, "Biology of Angiogenesis and Invasion in Glioma," Neurotherapeutics, vol. 6, no. 3, pp. 447-457, 2009.

[81] C. Calabrese, H. Poppleton, M. Kocak et al., "A perivascular niche for brain tumour stem cells," Cancer Cell, vol. 11, no. 1, pp. 69-82, 2007.

[82] R. J. Gilbertson and J. N. Rich, "Making a tumour's bed: glioblastoma stem cells and the vascular niche," Nature Reviews Cancer, vol. 7, no. 10, pp. 733-736, 2007.

[83] T. Borovski, J. J. C. Verhoeff, R. Ten Cate et al., "Tumor microvasculature supports proliferation and expansion of gliomapropagating cells," International Journal of Cancer, vol. 125, no. 5, pp. 1222-1230, 2009.

[84] E. Ruoslahti, "Specialization of tumour vasculature," Nature Reviews Cancer, vol. 2, no. 2, pp. 83-90, 2002.

[85] R. D. Folkerth, "Histologic measures of angiogenesis in human primary brain tumors," Cancer Treatment and Research, vol. 117, pp. 79-95, 2004.

[86] N. Ferrara, "Binding to the extracellular matrix and proteolytic processing: two key mechanisms regulating vascular endothelial growth factor action," Molecular Biology of the Cell, vol. 21, no. 5, pp. 687-690, 2010.

[87] M. C. Tate and M. K. Aghi, "Biology of angiogenesis and invasion in glioma," Neurotherapeutics, vol. 6, no. 3, pp. 447457, 2009. 

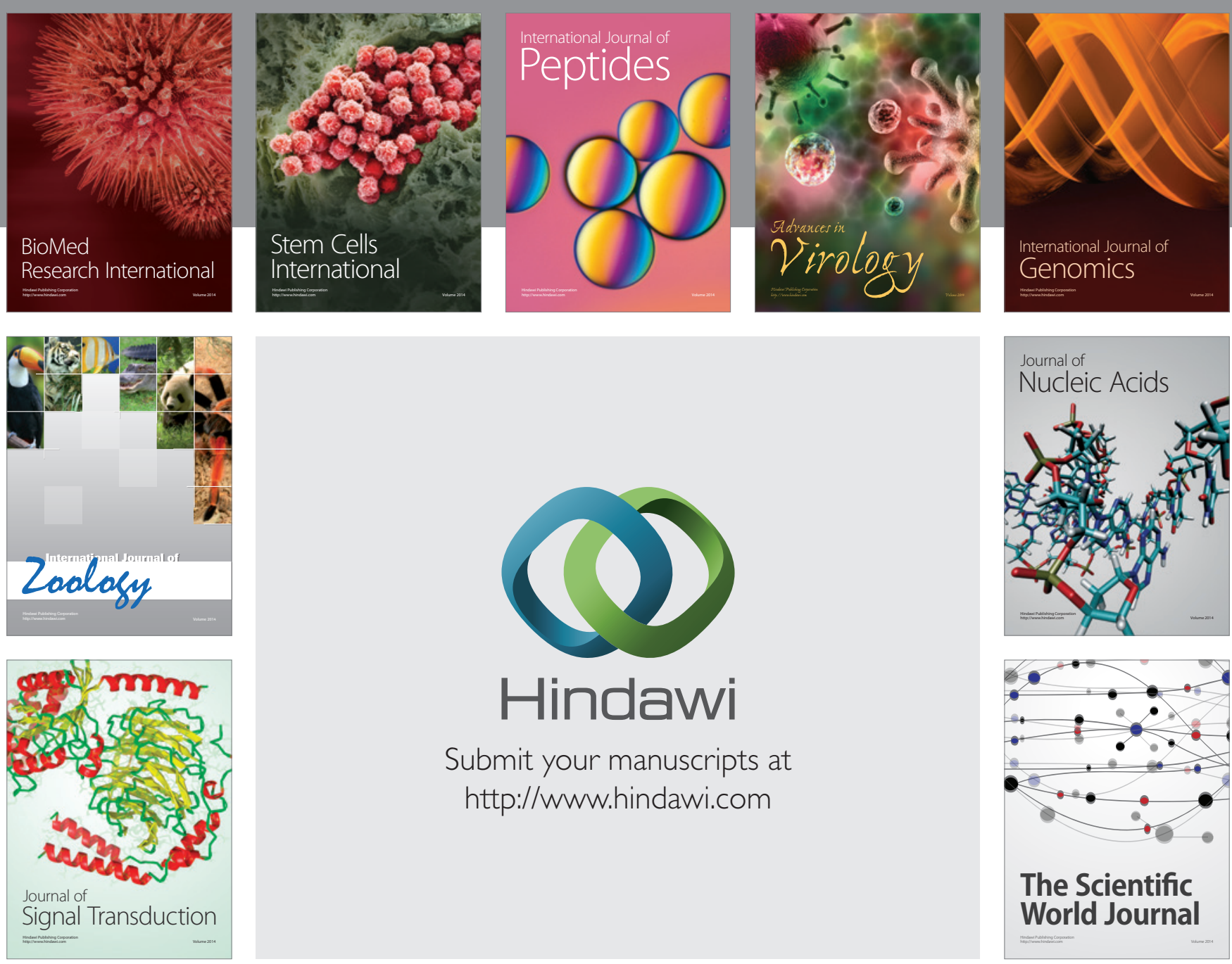

Submit your manuscripts at

http://www.hindawi.com
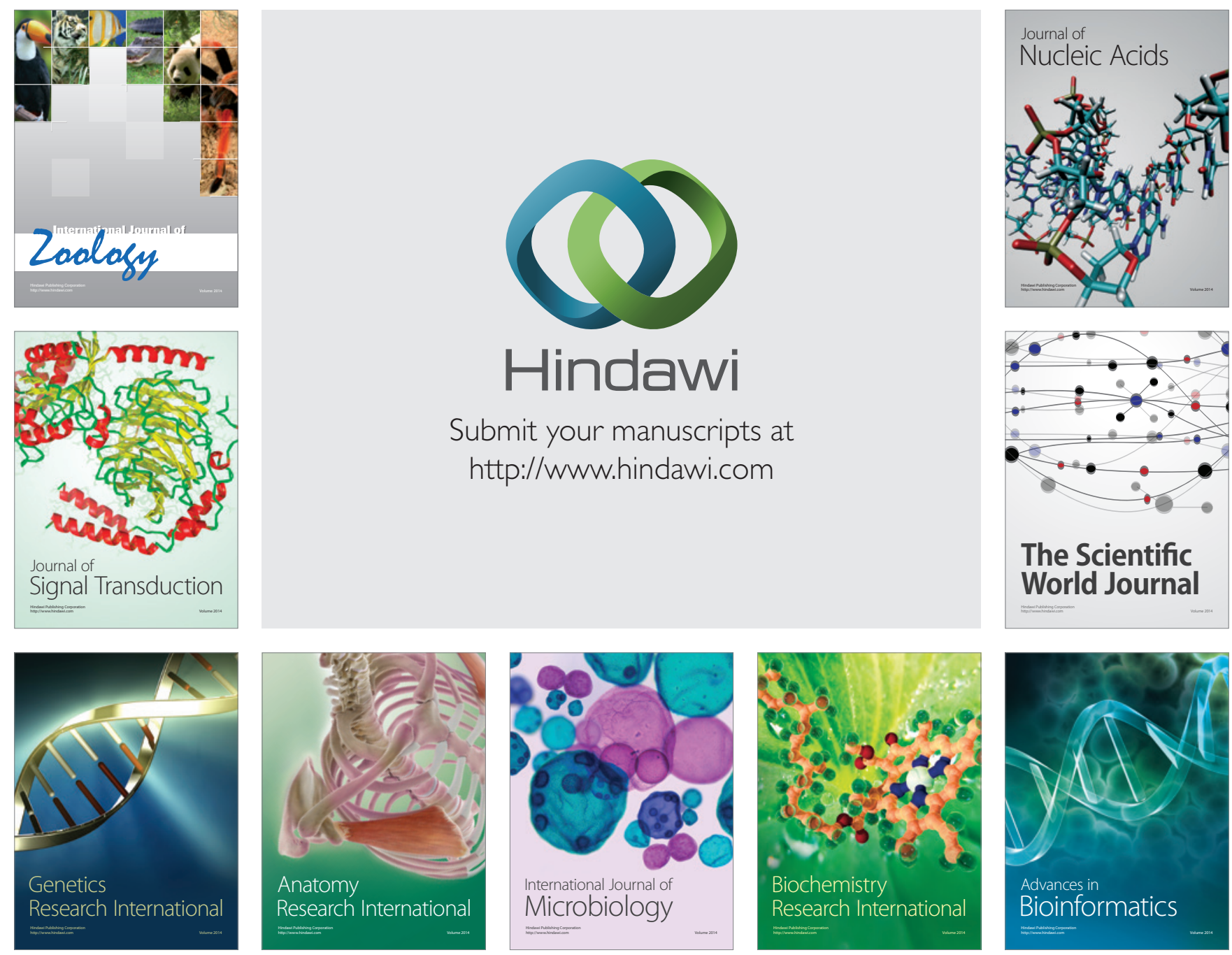

The Scientific World Journal
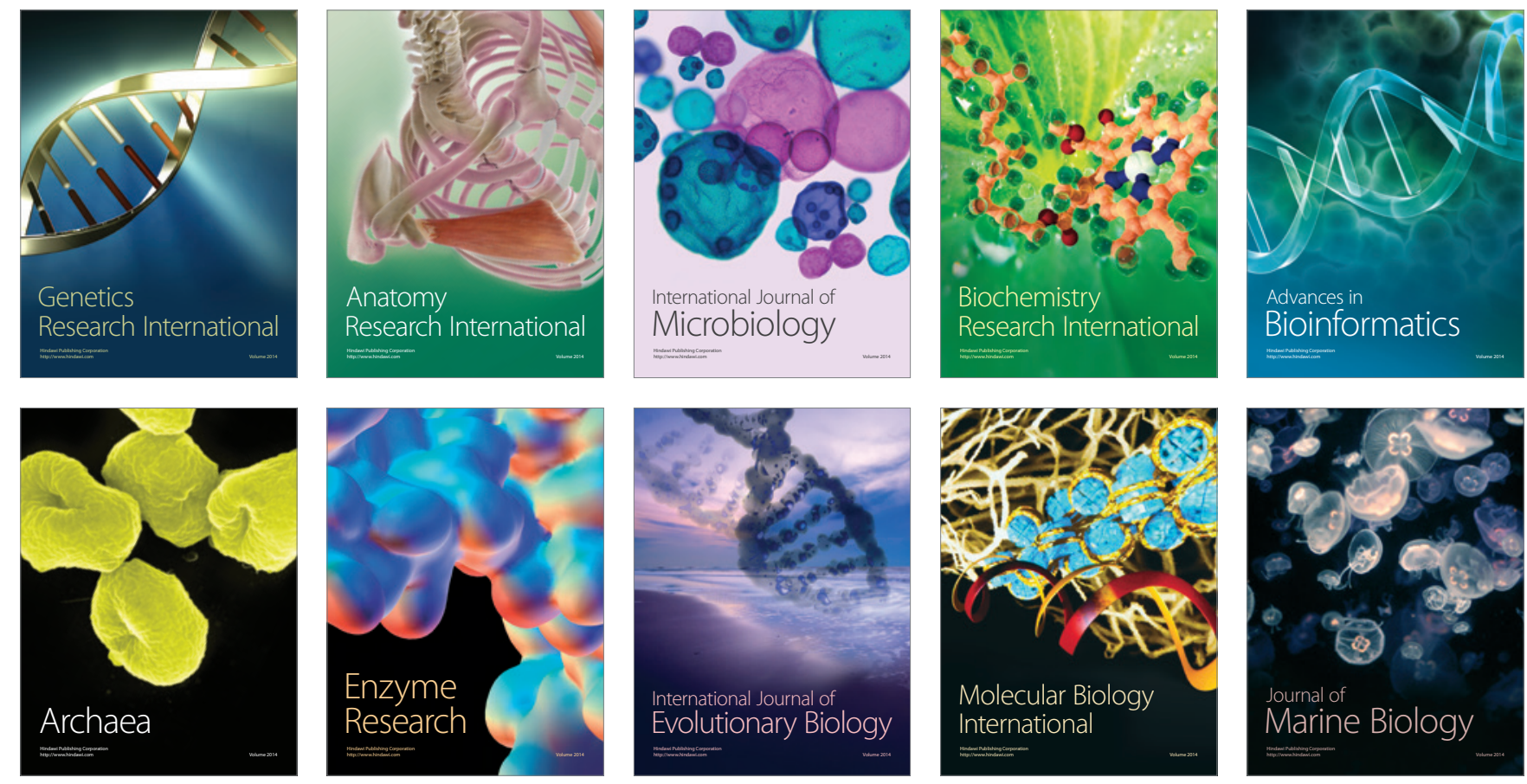